\title{
Simple Method of Record Base Fabrication for a Bar-Retained Implant Overdenture
}

\author{
Won-suk Oh, DDS, MS ${ }^{1} \&$ Ju-mi Park, DDS, MS, $\mathrm{PhD}^{2}$ \\ ${ }^{1}$ Department of Biologic \& Materials Sciences, University of Michigan School of Dentistry, Ann Arbor, MI \\ ${ }^{2}$ Department of Prosthodontics, Chonbuk National University School of Dentistry, Jeonju, Republic of Korea
}

\begin{abstract}
Keywords
Record base; cast mounting; implant

connecting bar; implant-retained overdenture.

Correspondence

Ju-mi Park, Chonbuk National University

School of Dentistry, Dept. of Prosthodontics, 664-14 Deokjin-dong 1-ga, Deokjin-gu,

Jeonju, Jeonbuk 561-756, Republic of Korea.

E-mail: jmpark@jbnu.ac.kr
\end{abstract}

The authors deny any conflicts of interest.

Accepted March 4, 2012

doi: 10.1111/j.1532-849X.2012.00893.x

\begin{abstract}
A record base should be stable and accurately transferable from the cast to the mouth. This article describes a simple and practical method of fabricating a record base for mounting a master cast used to fabricate an implant connecting bar for an implantretained overdenture.
\end{abstract}

A record base must be stable and transferable from the cast to the mouth for an accurate registration of an edentulous maxillomandibular relationship. ${ }^{1,2}$ However, a master cast made for an implant connecting bar to retain an implant-retained overdenture (IOD) does not precisely represent a patient's intraoral situation, because of the removal of healing abutments when making an implant-level impression. ${ }^{3,4}$

Healing abutments are, in general, retained on implants until intraoral placement of a bar-retained IOD. When making a final impression for the bar, the abutments are removed from implants, replaced with impression transfer copings, and repositioned intraorally after completion of the impression procedure. Thus, this master cast does not feature dome-shaped configurations of healing abutments extending above the ridge, but rather the platforms of implant replicas. 5,6

Several authors have introduced record base fabrication methods to address the disparity in the presentation of the cast to the mouth resulting from intraoral presence of healing abutments. ${ }^{5,6}$ One method requires healing abutments to be connected to implant replicas positioned in the master cast to match the patient's oral presentation. ${ }^{5}$ This procedure may enhance intraoral stability of the record base by gaining additional support from healing abutments placed on implants. Another method requires removal of healing abutments from implants prior to intraoral placement of a record base adapted against the platform of implant replicas in a master cast. This record base is often secured to implants by means of transfer copings or sleeves to enhance its stability. ${ }^{6}$ However, these procedures require additional parts of implants to be procured and close attention to detail when involving multiple dimensions of implants and healing abutments. In addition, prolonged removal of healing abutments may risk collapse of the periimplant gingiva while proceeding with multiple insertions and removals of the record base.

This article describes a practical method for fabrication of a record base for mounting a master cast, which is then used to fabricate an implant connecting bar to retain an IOD. This procedure makes the record base transferable from the cast to the mouth and keeps the laboratory and clinical procedures simple by eliminating additional parts of implants and multiple procedures of removal and replacement of healing abutments in the mouth.

\section{Procedure}

1. Using a stock impression tray, make a preliminary impression of an edentulous arch presenting with healing abutments placed on implants with an irreversible hydrocolloid impression material (Jeltrate; Dentsply Caulk, Milford, DE). Generate a study cast by pouring the impression in Type III dental stone (Microstone; Whip Mix Corp, Louisville, KY; Fig 1).

2. Block out undercuts on the study cast using baseplate wax (TruWax; Dentsply International Inc, York, PA). Adapt visible light-cured material (Triad Denture Base Material; Dentsply International, Inc.) against the representation of 


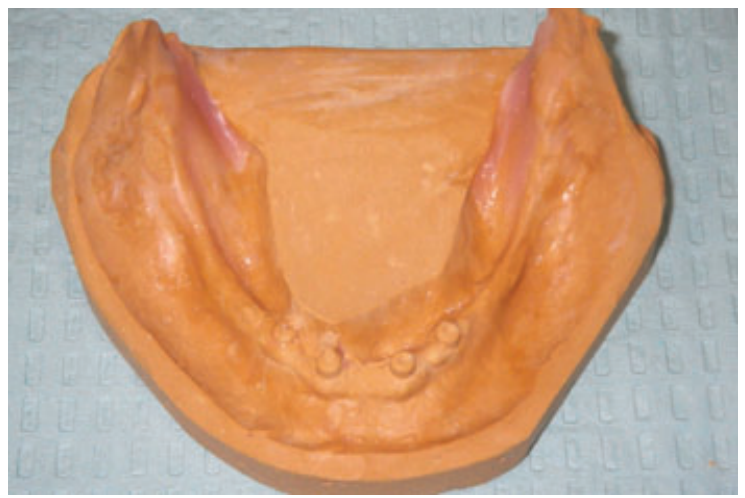

Figure 1 Study cast presenting dome-shaped configurations of healing abutments.

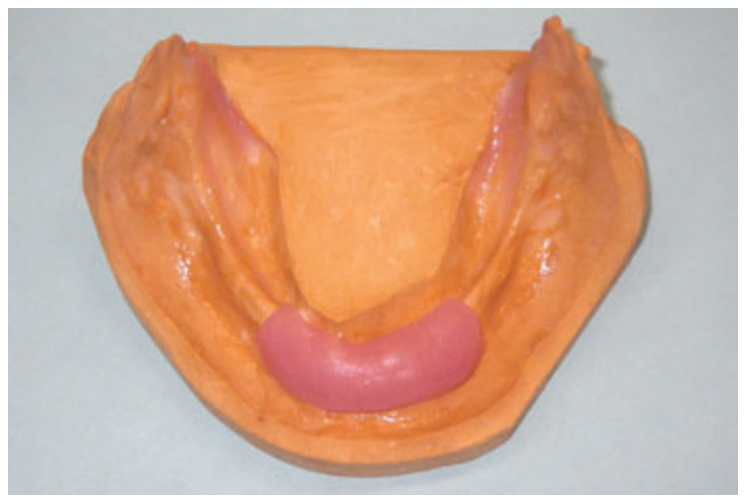

Figure 2 Sectional record base adapted against presentation of healing abutments in the implant sites of study cast.

healing abutments and extend it to cover approximately 2 to $3 \mathrm{~mm}$ of the facial and lingual ridge slopes on the implant sites (Fig 2).

3. Polymerize the record base material (Triad Denture Base Material) in a light chamber (Triad 2000 Curing Unit; Dentsply International, Inc.) for 2 minutes under visible light with a wavelength of $475 \mathrm{~nm}$ and a light intensity of $90 \mathrm{~mW} / \mathrm{cm}^{2}$. Separate the partially polymerized sectional record base from the study cast and set it aside.

4. Fabricate a custom tray using visible light-cured material (Triad VLC Custom Tray Material; Dentsply International, Inc.) on the same study cast for a pickup procedure of impression transfer copings. Make a final impression of the edentulous arch using an open tray technique ${ }^{7}$ to generate a master cast in Type IV dental stone (Silky Rock; Whip Mix Corp) (Fig 3).

5. Place the partially polymerized sectional record base on the master cast as reference to the ridge slope in the implant sites and secure it with fingers against the cast by joining additional visible light-cured material (Triad Denture Base Material) adapted on the edentulous area of the ridge (Fig 4). Polymerize the entire record base material (Triad Denture Base Material) in a light chamber (Triad 2000 Curing Unit) for 6 minutes and trim it using a tungsten carbide bur (E-cutter; Brasseler USA, Savannah, GA).

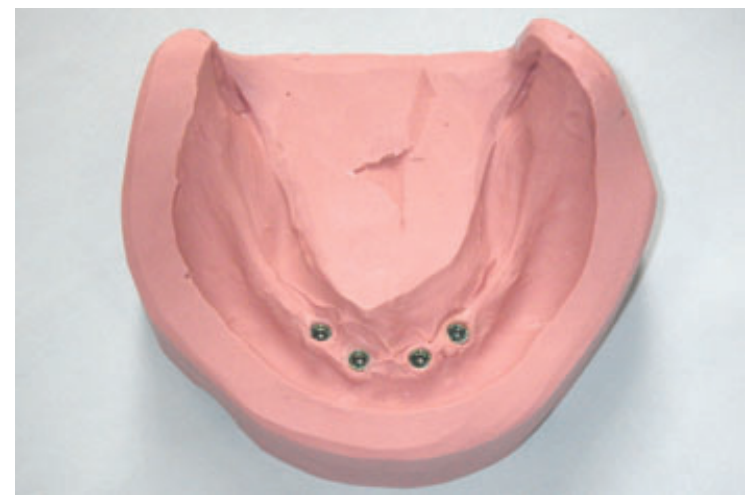

Figure 3 Master cast presenting platforms of implant replicas.

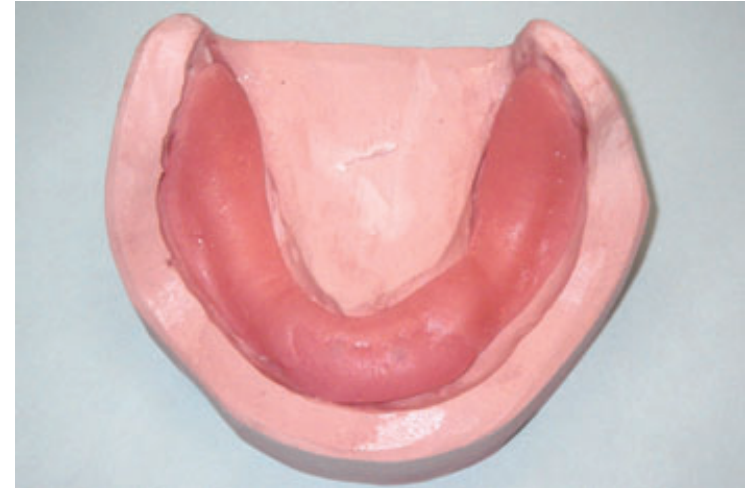

Figure 4 Completed record base adapted on edentulous area of ridge joining sectional record base on master cast.

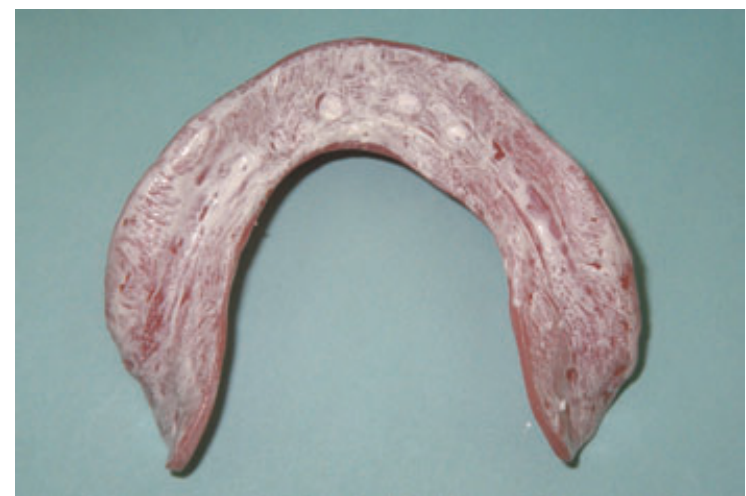

Figure 5 Intraoral fit of record base as indicated by pressure-indicating paste.

Build up an occlusion rim using baseplate wax (TruWax) on the completed record base.

6. Evaluate intraoral fit of the record base using pressureindicating paste (Mizzy Inc, Cherry Hill, NJ) against healing abutments and the edentulous area of the ridge and adjust it as needed using a tungsten carbide bur (E-cutter; Fig 5). Proceed to register the edentulous maxillomandibular relationship to mount the master cast used to fabricate an implant connecting bar for an IOD. 


\section{Discussion}

The fabrication of an IOD retained by an implant connecting bar requires multiple impression and mounting procedures for study and master casts. The first mounting of a master cast would be to design the bar conforming to a diagnostic arrangement of denture teeth. A secondary final impression is made over the cast bar fitted intraorally to generate another master cast for an IOD, where the cast mounting is accomplished by means of a newly fabricated record base assisted by the bar. Thus, the first mounting of a master cast for the bar can be considered as a tentative procedure prior to orienting a final master cast for the IOD.

This method of record base fabrication involves two-stage procedures where the anterior portion of the base fits against healing abutments, and the posterior portion conforms to the edentulous area of the ridge. When fitting the record base in the mouth, the stability of the record base should be ensured by eliminating binding areas against the healing abutments. Thus, the accuracy of this record of maxillomandibular relationship will be within the range of compressibility of the edentulous ridge under the record base.

\section{Summary}

Stability of a record base plays a significant role in registering an accurate relationship of edentulous maxilla and mandible.
The record base also should be transferable from the cast to the mouth; however, a master cast used to fabricate an implant connecting bar for an IOD does not present dome-shaped configurations of healing abutments when the impression is made at implant level. This method of record base fabrication simplifies clinical and laboratory procedures for mounting a master cast for an implant connecting bar to retain an IOD, without losing the accuracy of the record base fit.

\section{References}

1. Oh W, May KB: Two-stage technique for optimum fit and stability of light-polymerized record bases. J Prosthet Dent 2008;99:410-411

2. Akerly WB: Recording jaw relationships in edentulous patients. Dent Clin North Am 1996;40:53-70

3. Loney RW: Stabilized record bases for implant-retained overdentures. J Prosthet Dent 1994;72:16-18

4. Keith SE, Guillen GE: Enhanced stability for implant-supported overdenture record bases. J Prosthet Dent 1998;79:359-360

5. Rungcharassaeng K, Kan JY: Fabricating a stable record base for completely edentulous patients treated with osseointegrated implants using healing abutments. J Prosthet Dent 1999;81:224-227

6. Nimmo SS, Nimmo A: Stabilized record base for implant treatment. J Prosthodont 2010;19:586-588

7. Windhorn RJ, Gunnell TR: A simple open-tray implant impression technique. J Prosthet Dent 2006;96:220-221 